\title{
Erratum to: Reappraisal of treatment-induced renal dysfunction in patients receiving antiangiogenic agents in phase I trials
}

\author{
Antonin Levy • Laurence Albiges-Sauvin • Christophe Massard • Hassan Izzedine • \\ Stéphane Ederhy $\cdot$ Ratio Bahleda $\cdot$ Carlos Gomez-Roca $\cdot$ Cyrus Chargari \\ Isabelle Brocheriou-Spelle • Jean-Charles Soria
}

Published online: 3 January 2012

(C) Springer Science+Business Media, LLC 2011

Erratum to: Invest New Drugs

DOI 10.1007/s10637-011-9671-z

We would like to correct one Author's name on our manuscript:

-Stéphane Ederhy (instead of Stéphane Edhery)

The online version of the original article can be found at http://dx.doi. org/10.1007/s10637-011-9671-z.

A. Levy · L. Albiges-Sauvin · C. Massard · S. Ederhy •

R. Bahleda $\cdot$ C. Gomez-Roca $\cdot$ J.-C. Soria $(\bowtie)$

SITEP (Service des Innovations Therapeutiques Precoces),

Department of Medicine, Institut Gustave Roussy,

Paris XI University,

114 rue Edouard Vaillant,

94805 Villejuif, France

e-mail: soria@igr.fr

\author{
A. Levy $\cdot$ C. Chargari \\ Inserm 1030, Institut Gustave Roussy, Paris XI University, \\ Villejuif, France \\ H. Izzedine \\ Department of Nephrology, AP-HP, Hôpital Pitié Salpetrière, \\ Paris, France \\ I. Brocheriou-Spelle \\ Department of Pathology, AP-HP, Hôpital Pitié Salpetrière, \\ Paris, France

